\title{
I 30 Cardiac diastolic function evaluation using a novel method for quantification of cine myocardial deformation analysis (cMDA) based on non-rigid registration

\author{
Himanshu Gupta*1, Wei Feng ${ }^{2}$, Steven G Lloyd ${ }^{1}$, Thriveni Sanagala1,
} Louis J Dell'italia ${ }^{1}$ and Thomas S Denney Jr${ }^{2}$
}

Address: ${ }^{1}$ University of Alabama at Birmingham, Birmingham, AL, USA and ${ }^{2}$ Auburn University, Auburn, AL, USA

* Corresponding author

from I th Annual SCMR Scientific Sessions

Los Angeles, CA, USA. I-3 February 2008

Published: 22 October 2008

Journal of Cardiovascular Magnetic Resonance 2008, IO(Suppl I):A255 doi:I0.I I86/I532-429X-I0-SI-A255

This abstract is available from: http://jcmr-online.com/content/I0/SI/A255

(C) 2008 Gupta et al; licensee BioMed Central Ltd.

\section{Introduction}

Quantitative evaluation of myocardial strains is important in various cardiac diseases. Tagged cardiac MRI with either image or spectral-based analysis is used for such purposes in a few centers. There are many disadvantages, however, that preclude widespread use of tagging or other specialized techniques such as DENSE for routine practice. One major disadvantage of tagging is that diastolic function is difficult to measure due to tag fading. Here we describe the use of a novel method for quantification of cine myocardial deformation analysis (cMDA) based on non-rigid registration to evaluate diastolic function in mitral regurgitation (MR) and hypertensive (HTN) heart disease, using standard cine MRI.

\section{Methods}

All procedures were performed per the institutional guidelines after obtaining informed consent. Standard Cardiac MRI views were obtained on a GE 1.5 T system using ECG gated steady state free precession (SSFP) technique with following parameters: slice thickness $8 \mathrm{~mm}$, Field-of-view $40 \times 40 \mathrm{~cm}$, scan matrix $256 \times 128$, flip angle $45^{\circ}, \mathrm{TR} / \mathrm{TE}$ $=3.8 / 1.6 \mathrm{~ms}$, temporal resolution $\leq 50 \mathrm{~ms}$. 38 normal (nrm), 106 HTN and 45 MR were imaged. Non-rigid image registration techniques were used to measure inplane myocardial deformation between imaged cardiac phases in a set of mid-ventricular short-axis slices. These deformations were used to compute a time series of diastolic strains and strain rates.

\section{Results}

HTN group demonstrated concentric hypertrophy whereas MR group demonstrated eccentric left ventricular remodeling. Important differences in the diastolic circumferential and radial relaxation rates were noted in the 3 groups. HTN had a lower early circumferential relaxation rate (corresponding to the $\mathrm{E}$ wave of early diastole) with delayed peak early circumferential relaxation rate compared to MR (Fig. 1, p < 0.05). Late circumferential relaxation rate (corresponding to the A wave of late diastole) was greater in HTN as compared to MR (Fig. 2, p < 0.05). Although the early radial relaxation rate were similar in 3 groups, time to peak early radial relaxation rate in HTN was longer than MR or nrm group. The late diastolic radial relaxation rate was greater in HTN as compared to the other groups (Fig. 3, p < 0.05).

\section{Conclusion}

Important differences in the diastolic properties of two distinct clinical groups (MR and HTN) are noted that can be evaluated using CMDA methodology. cMDA analysis can be applied to routine non-tagged functional cardiac images and is relatively easy to implement. Therefore cMDA methodology holds great promise in its application to cardiac mechanics under different clinical conditions. 

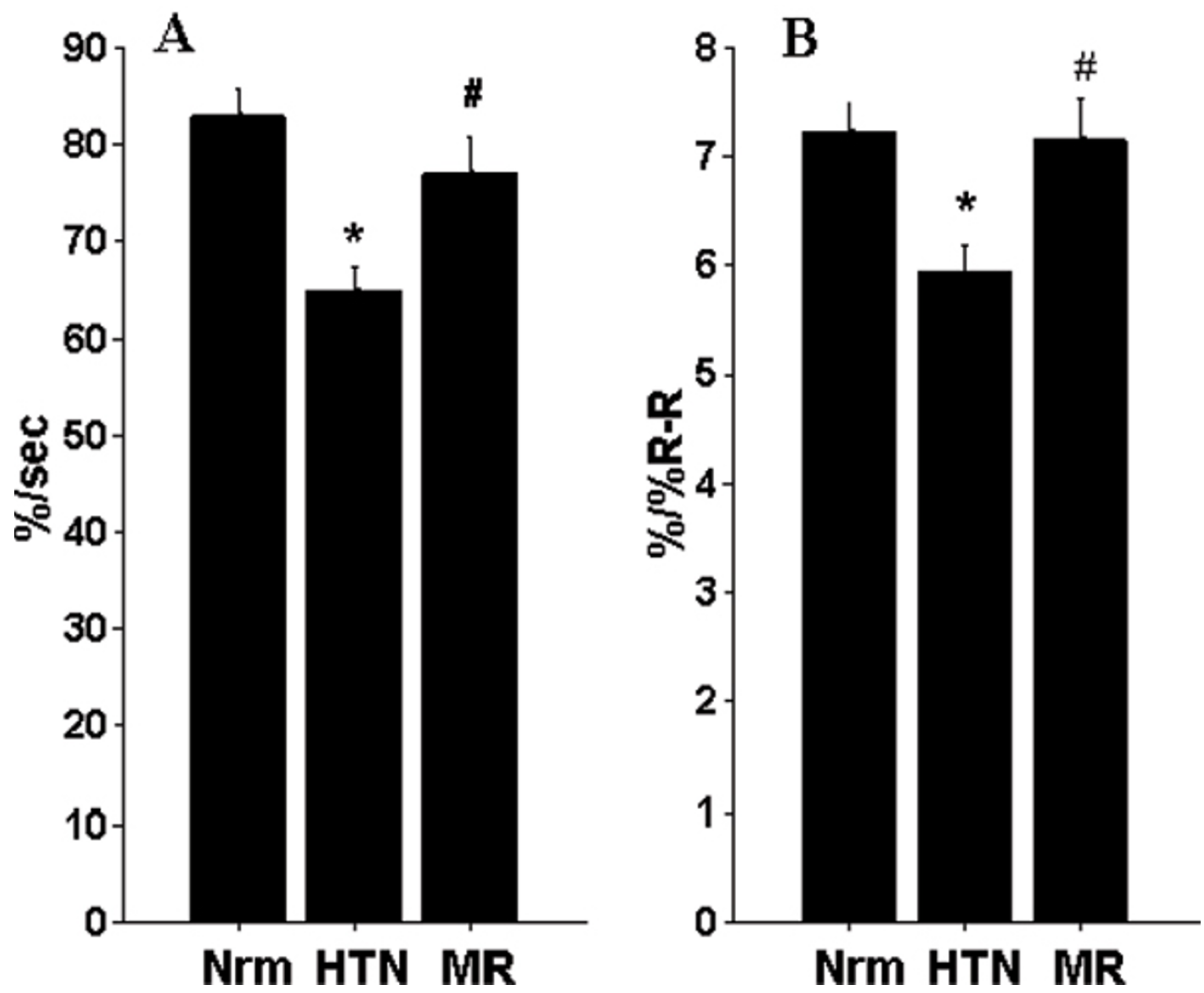

Figure I

Early diastolic circumferential relaxation rates calculated by cMDA technique A) Expressed as \%/sec; B) Expressed as \%/\%R-R interval; *p $<0.05$ vs. normal; \#p < 0.05 vs.HTN. 

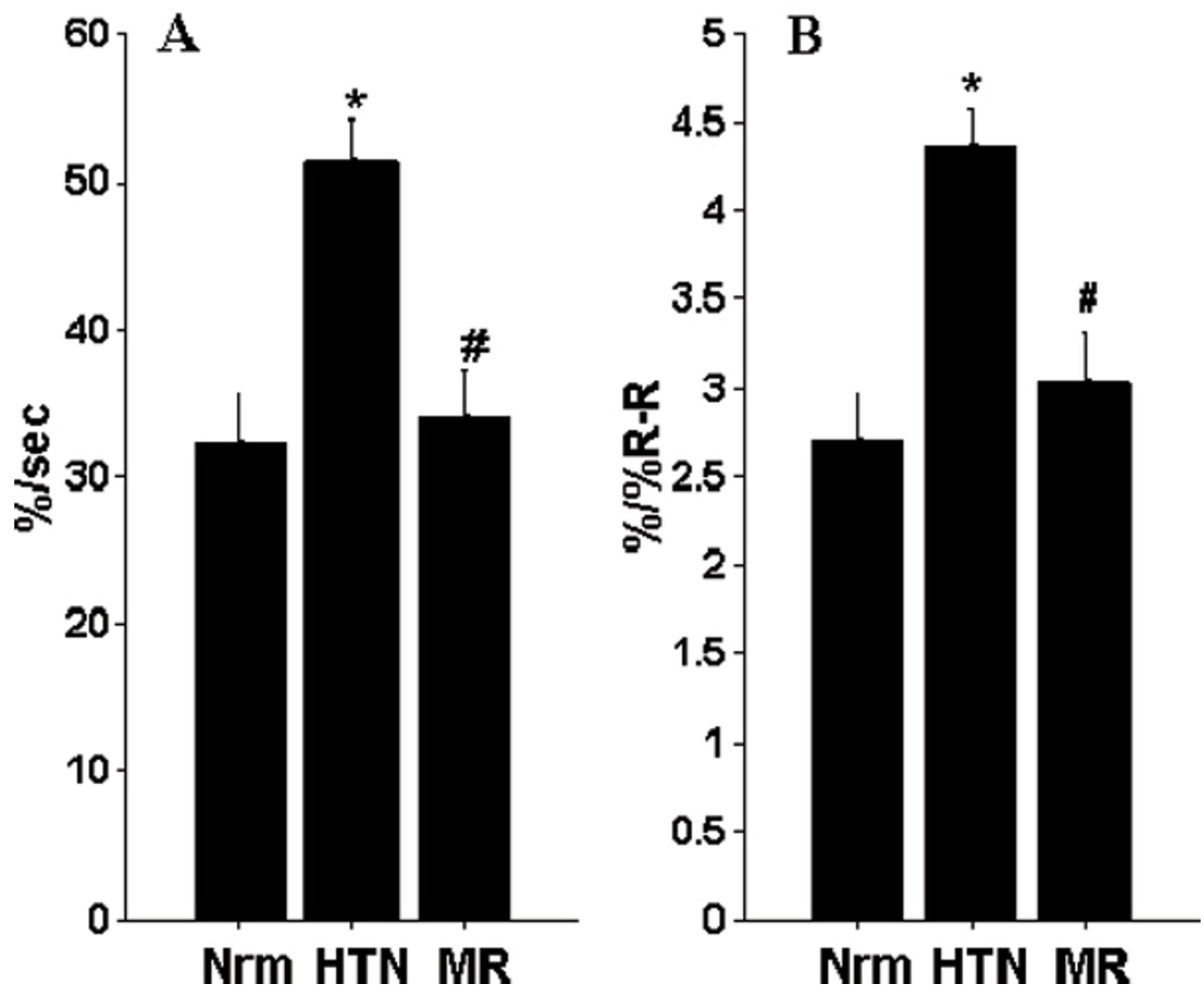

Figure 2

Late diastolic circumferential relaxation rates calculated by cMDA technique A) Expressed as \% sec; B) Expressed as \%/\%R-R interval; ${ }^{*} \mathrm{p}<0.05$ vs. normal; \#p $<0.05$ vs. HTN. 

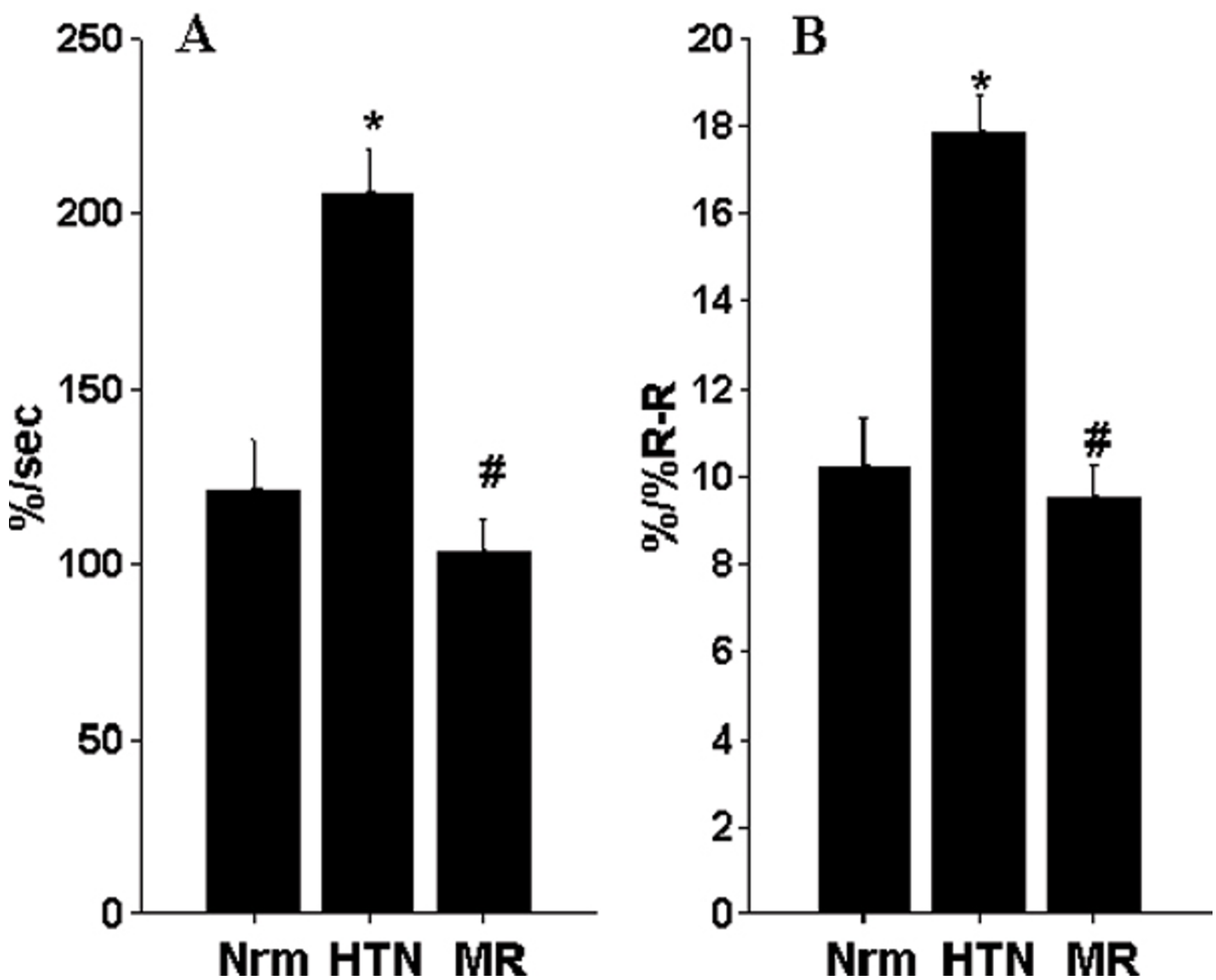

Figure 3

Late diastolic radial relaxation rates calculated by cMDA technique A) Expressed as \% sec; B) Expressed as \%/\%R-R interval; *p $<0.05$ vs. normal; \#p < 0.05 vs. HTN. 